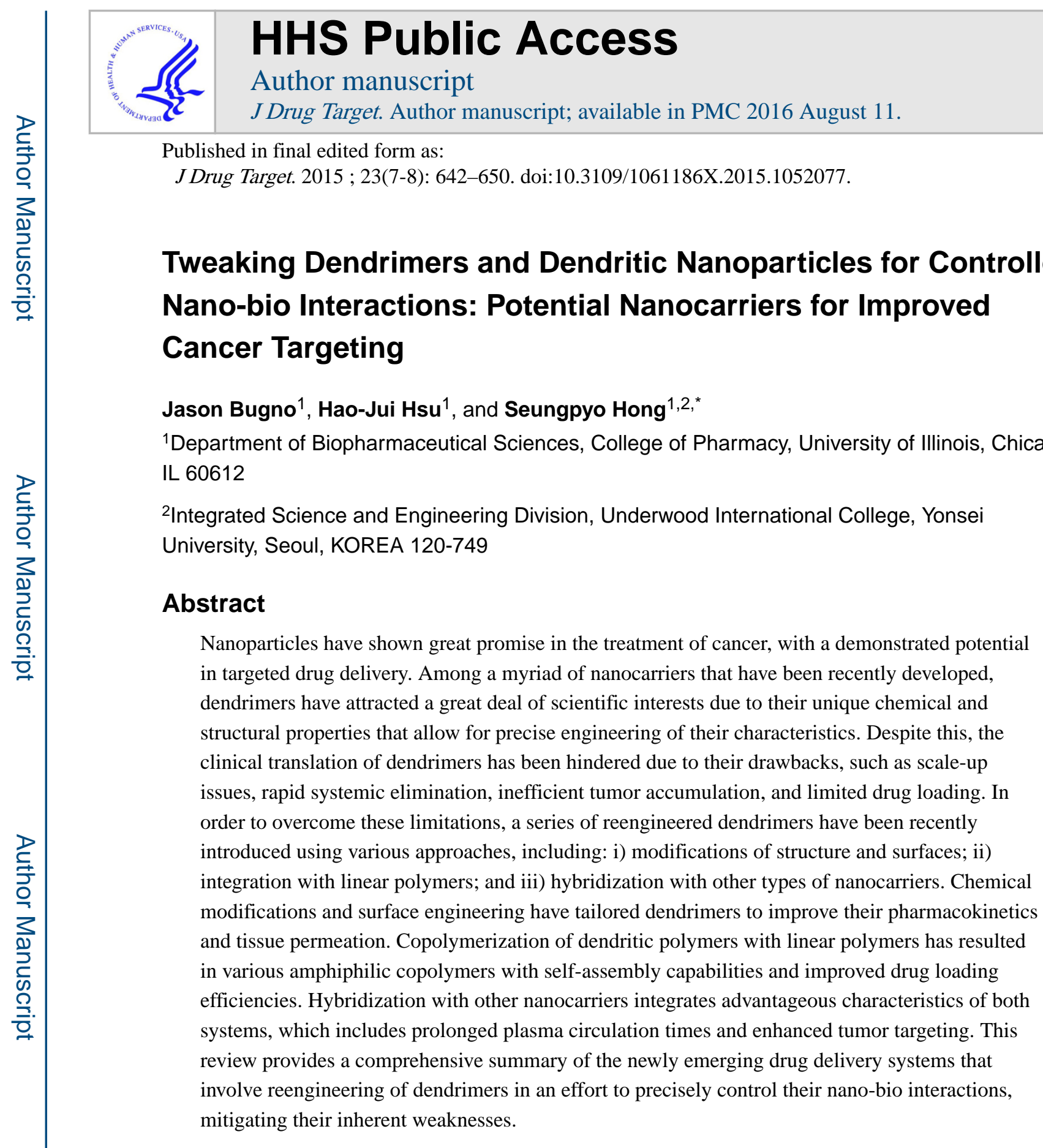

\title{
1.0 Introduction
}

With greater than 1.6 million newly diagnosed cases projected each year, cancer and other malignant diseases represent one of the greatest burdens on patients and the modern healthcare system.(1) This makes the treatment of cancer an area with a high demand for innovation and technological advances. Among these advances, nanoparticles (NPs) have garnered significant attention because their properties can be engineered and fine-tuned to enable the selective delivery of drugs, biologics, and imaging agents.(2) However, NPs are

\footnotetext{
*All correspondence should be addressed to: Prof. Seungpyo Hong, Ph.D., Department of Biopharmaceutical Sciences, College of Pharmacy, The University of Illinois at Chicago, 833 S. Wood St. Rm 335, Chicago, IL 60612.
} 
faced with significant biological barriers that often prevent them from achieving clinically significant tumor targeting efficacy, which includes the undesirable adsorption of circulating proteins and immune complexes, the limited permeation of endothelial layers and tumor tissue, and the selective permeability of cell membranes. $(3,4)$ As our understanding of the interactions on the nano-bio interface continues to expand, a number of novel techniques and NP design strategies are under investigation to overcome these barriers.(5)

Polymeric NPs provide unique opportunities for tailoring their biological interactions and engineering new functionalities, all while presenting one of the most biocompatible NP platforms. Poly(lactic-co-glycolic acid), poly(lactic acid), and poly(ethylene glycol) have been most commonly utilized as they are approved by the FDA for human uses. $(6,7)$ Several of the major advances in polymeric NPs were pioneered by the honoree of this issue, Prof. Robert Langer.(8-10) One of the companies he co-founded, BIND Therapeutics, has a leading candidate, BIND-014, that is a $100 \mathrm{~nm}$ polymeric NP encapsulating the anti-cancer drug docetaxel, which has shown great promise in clinical tumor treatment.(11) This pioneering work of Prof. Langer, along with his early contribution to the fields of drug delivery and polymer sciences, has led to a wide range of polymers being investigated for use in biomedical applications. Polymers for use in biomedical applications can be prepared over a wide array of molecular architectures, as illustrated in Figure 1.(12,13) For instance, conventional linear polymers are prepared from the linear growth of chains and as such can adopt various molecular conformations. In contrast, hyperbranched polymers are prepared by the repetitive branching of monomeric units, and can be used for generating polymers with a highly dense presentation of surface groups. Star polymers are formed from the growth of linear chains initiated with a multifunctional, central core. Among these architectures are dendrimers and dendrons, small ( $<10 \mathrm{~nm}$ in diameter) hyperbranched polymers that grow radially from a core molecule, presenting one of the most promising nanocarrier platforms.

Dendrimers and dendritic NPs are well-suited for biomedical applications due to their precisely controlled size, well-defined and near-monodisperse structure, and surface presentation of terminal functional groups.(14) However, despite decades of insightful in vitro and in vivo biomedical research, they still suffer from several limitations such as rapid systemic elimination, inefficient tumor targeting, and poor/unstable drug loadings (particularly of hydrophobic molecules) that have hindered their rapid clinical translation, resulting in only two clinical trials to date. In order to overcome these barriers, it has been found that targeted, rational engineering to the dendrimer structure and their hybridization with other nanocarriers can be viable methods for controlling their biological interactions. (15) Some of these have included surface modifications, preparation of Janus, or two-faced dendrimers, alterations of the molecular architecture by integrating linear polymers, and hybridization with other NPs. This review will provide a comprehensive overview of these designs, focusing on the rational design and tweaking of dendritic nanomaterials to control their nano-bio interactions and tumor targeting behaviors. We will also discuss approaches hybridizing the dendritic architecture with other nanocarriers. This overview will encompass recent insights into the mechanisms underlying the interactions of dendritic polymers with 
biological systems and how these materials can be engineered to overcome biological barriers to achieve selective delivery of therapeutic agents.

\subsection{Dendrimers and Dendritic Architecture}

\subsection{Dendrimer Synthesis}

Dendrimers and dendritic polymers are unique in their synthesis compared to other macromolecules in that they are grown radially in a tightly controlled manner through sequential activation and condensation reactions. $(16,17)$ Each subsequent step results in the next generation $(\mathrm{G})$. While several synthetic strategies have been proposed, two of the most commonly used methods are the divergent and convergent methods. When the dendrimer is grown radially from a multifunctional core, the method is referred to as divergent synthesis. $(18,19)$ Divergent synthesis can confer distinct advantages in dendrimer preparation. For instance, the dendrimer surface can be easily modified during the final reaction step, endowing specific surface functionalities. Although divergent synthesis is the more widely adopted method for dendrimer preparation, it is limited by the presence of branching defects in the final products, particularly at higher generations. In contrast, convergent approaches couple individually synthesized dendrons to a multifunctional core, forming a dendrimer.

(20) While convergent synthesis is great for producing smaller generation dendrimers with a well-defined structure, as the size increases steric hindrance between the large dendrons can block the formation of fully spherical dendrimers. Nonetheless, the purification of dendrimers prepared this way is easier than the divergent method due to larger differences between the final products and smaller starting materials. Additionally, the convergent strategies allow for the preparation of dendrimers composed of different dendrons, a common approach in the synthesis of Janus, or two-faced, dendrimers that will be discussed in a subsequent section.

Although dendrimer synthesis makes for polymers with low polydispersities, their scaling ability and purity is limited by branching defects and heterogeneous surface distributions. Uncompleted reaction steps can cause prematurely terminated arms, and unremoved starting materials can begin new, lower generation dendrimers. For instance, van Dongen et al. have demonstrated that G5 poly(amidoamine) (PAMAM) dendrimers purchased from commercial suppliers contain only 93 arms on average, as opposed to the theoretically predicted 128.(21) Within the material, major structural defects, including multimer species and trailing generations, were identified and characterized. Similarly, due to inherent statistical effects, surface modification of dendrimers with functional groups make the preparation of welldefined materials with precise numbers of ligands difficult. Only recently have researchers begun to prepare dendrimers with distinct numbers of functional groups.(22, 23) Moreover, these advances have led to better elucidation of the mechanism involved in the binding of folic acid (FA)-conjugated dendrimers to the folate binding protein, suggesting that more precise dendrimer-ligand distributions are needed to define the surface interactions of dendrimers with biological systems.(24) 


\subsection{Features of the Dendritic Architecture}

The radial growth, well-defined molecular architecture, and globular structure of dendrimers confer them with unique physicochemical characteristics.(25) At each step of generational growth, the molecular weight and number of surface groups of the dendrimer double. For example, while G2 PAMAM dendrimers prepared with an ethylenediamine core have 16 functional groups, G3 dendrimers have 32, and G7 dendrimers present a theoretical 512 surface groups. Ranging in size from only a few, to tens of nanometers, dendrimers have a high ratio of surface groups-to-volume. Furthermore, dendrimers have highly flexible structures, and smaller generations tend to have amorphous structures that adopt spherical, globular conformations.(26) Moderately sized materials, such as G5 PAMAM dendrimers are similar in size and shape to the protein hemoglobin.(17) Larger PAMAM dendrimers, beginning with $\mathrm{G} 7$ and going upwards, have a higher ratio of internal to external surface areas and tend to adopt more rigid conformations.(27) Another unique aspect of dendrimers is their rheological properties. Unlike conventional linear polymers that typically exhibit high viscosities at high concentrations due to the chain entanglement, the globular structure of dendrimers minimizes chain entanglement, resulting in little-to-no increases in viscosity even at very high concentrations. $(28,29)$ All in all, the dendritic structure can be precisely engineered to control particle size, surface, and physicochemical/rheological properties, making them valuable for use in a variety of applications.

\subsection{Limitations to the Use of Dendritic Polymers}

Despite their advantageous characteristics and decades of biomedical research, dendrimers have still not seen rapid clinical translation as nanocarriers due to several limitations. For example, it is difficult to obtain consistent loading efficiencies and controlled drug release patterns. Most commonly, drugs are either chemically conjugated to the dendrimer surface or physically encapsulated within their internal void volume.(30) Chemical conjugation can result in higher loadings (typically up to 10\%) and controlled, stimuli-responsive release. For instance, Satsangi and co-authors were able to deliver paclitaxel specifically to tumors over-expressing cathepsin B using a cathepsin B cleavable linkage, resulting in higher reductions in tumor size compared to the free drug.(31) However, chemical conjugation is inherently limited by the availability of drugs that can be chemically modified without losing their potency, and conjugation of too many drug molecules per dendrimer causes a significant increase in the polydispersity index of the dendrimer. Alternatively, drugs can be physically encapsulated within the internal void volume of the dendrimer; however, this often leads to erratic release profiles, with large initial bursts. When methotrexate (MTX) was encapsulated within dendrimers, greater than $70 \%$ of MTX was released within the first $2.5 \mathrm{~h}$, much more rapidly than the drug-dendrimer conjugates. (32)

The use of cationic dendrimers is often associated with significant toxicological issues. Although they are particularly useful in delivery of genes and oligonucleotides, they also cause non-specific cell uptake and toxicities. $(33,34)$ It has been reported that the dense surface charge of large cationic dendrimers causes them to interact strongly with anionic lipid bilayers via electrostatic interactions, forming nanoscale holes in the cell membranes and inducing significant cellular toxicity, most notable at higher generations of PAMAM dendrimers. Positively charged dendrimers have been associated with toxicities at 
concentrations as low as $12 \mu \mathrm{g} \mathrm{ml}^{-1}$, limiting their use in vivo. $(33,35)$ These findings suggest that surface modification of dendrimers is necessary for use in biological systems, particularly for drug delivery applications.

Of the greatest barriers associated with dendrimer use is their rapid systemic clearance and inadequate tumor accumulation. For instance, smaller PAMAM dendrimers (G2-G4) exhibit rapid renal clearance, and their larger counterparts are uptaken by the liver and spleen, organs of the reticuloendothelial systems (RES). $(36,37)$ This rapid elimination from plasma circulation, along with the fact that dendrimers are too small to take significant advantage of the EPR effect, can lead to inefficient tumor delivery and accumulation. One approach to increase tumor tissue localization is to use targeting ligands. The Baker group designed FAtargeted PAMAM dendrimers that were found in the tumor and liver tissue 4 days following injections into mice containing FR-overexpressing tumors, increasing the therapeutic response.(38) Nevertheless, non-targeted dendrimers accumulated significantly in the kidneys and the targeted ones in the liver, with less than $10 \%$ of the dose being accumulated at the tumor site. Alternatively, Wu and co-workers found that surface modification with oligoethylene can be used to enhance the tumor accumulation and permeability of dendrimers.(39) Although dendrimers conjugated with the anti-cancer drug gemcitabine had positive responses, more than $30 \%$ of the initial dose was still uptaken by the kidneys within $10 \mathrm{~min}$ after intravenous injection. Taken together, even with the significant advances of dendrimers, it is clear that dendrimer-based nanocarriers need to improve to achieve higher drug loadings with stable release patterns, controlled cellular interactions, and improved plasma circulation times with enhanced tumor accumulation.

\subsection{Approaches to Overcoming Drawbacks of Dendrimers}

To address the issues of dendrimers discussed above, a number of approaches for dendrimer modifications have been attempted to ultimately engineer highly efficient nanocarriers. In this review, among many promising approaches, we will focus on: i) chemical/synthetic modifications; ii) copolymerization with linear polymers; and iii) hybridization with other NPs. These design strategies can be used to overcome the limitations described above. For instance, the surface charge modification of dendrimers can be used to precisely tailor their cellular interactions. Janus dendrimers and dendrimer-linear copolymers exhibit enhanced drug and gene loadings compared to conventional dendrimers. Moreover, self-assembled dendrimer-linear copolymers take this one step further and can form stable structures with enhanced plasma circulation times and tumor targeting capabilities. Hybridizing the dendrimer with other nanocarriers can similarly overcome their poor circulation times and biodistributions, with the added benefit of maintaining their small size and enhanced tumor permeation.

\subsection{Chemical Modification to Dendrimers}

3.1.1 Charge Modifications-One of the easiest ways to engineer dendrimers and control their biological interactions is to chemically modify their surfaces. Dendrimers have been surface modified to contain a variety of charge types, imaging agents, therapeutic drugs, and targeting ligands. One of the most useful methods for engineering the biological 
interactions of dendrimers has been to change the surface charge. For instance, our group has demonstrated that the skin permeation of G2 PAMAM dendrimers can be manipulated by engineering their surface functionalities.(40) Neutralizing the dendrimer surface enhanced the permeation of the dendrimers through the stratum corneum, the outermost layer of the skin, through an extracellular pathway. In contrast, amine-terminated cationic dendrimers exhibited significant interactions with the dermal components, showing enhanced cellular internalization. Despite greater retention in the skin layers, skin permeation of the positively charged dendrimers was impaired. Similarly, the surface charge of dendrimers is intricately tied to their biodistributions following systemic injection. While non-specific interactions of cationic dendrimers with the vasculature decreased their systemic availability, carboxylated dendrimers displayed enhanced circulation times by 10-20 fold. $(35,41)$ These findings show that the surface charge of dendrimers plays a critical role in their interactions in vivo. Despite this, surface-modified dendrimers are still unable to carry significant amounts of drug molecules without the use of chemical conjugation, which requires the drug molecule to have a chemically reactive group.

3.1.2 Janus (Two-Faced) Dendrimers-Although conventional dendrimers have garnered great attention for biomedical applications over the last few decades, they are limited because surface ligands and modifications can only be distributed randomly on their surfaces. Alternatively, dendrimers can also be prepared to contain regions of chemically and structurally distinct groups. These dendrimers have been most often referred to as Janus dendrimers, referencing the Roman god often portrayed by having two opposing faces.(42) By linking dendrons with different compositions and surface modifications, Janus dendrimers can be prepared to present regionally distinct surface groups. Janus dendrimers also have potential to overcome the low drug loadings of hydrophobic cargos or oligonucleotides that limit conventional dendrimers.

Janus dendrimers can be synthesized using one of the three main methods.(42-45) The first method involves the conjugation of individual dendrons with complementary core functionalities. Alternatively, the second method utilizes the initial modification of one dendron with a multifunctional group, followed by the coupling with the second dendron. The third method polymerizes the second dendron from the core of the first using sequential activation and condensation reactions. These methods allow for the generation of dendrimers that have chemically distinct surface regions, and can allow for the hybridization of dendrimers of different compositions and distinctly different architectures. For instance, amphiphilic Janus dendrimers can form stable self-assembled structures via the formation of micelles in aqueous solutions. Not only does the self-assembled micelle formation increase the size to improve plasma circulation times, but can also allow the formation of stable hydrophobic cores that enhance drug loading efficiencies (8-10 wt\%).(46) While it incorporates this in the structure, the Janus dendrimers are also able to take advantage of the high surface coverage endowed by the dendritic architecture. Amphiphilic Janus dendrimers consisting of poly(L-lysine) (PLL) and saturated carbon chains are able to complex RNA through electrostatic interactions between the cationic dendrimer and anionic backbone of the oligonucleotide. $(47,48)$ The strong complexation, in addition to the hydrophobic side 
chains that enhanced interactions with the cell membranes, resulted in a drastically increased transfection efficiency of the polyplex.

Despite a number of advantageous aspects, Janus dendrimers are still limited by barriers that plague the use of more conventional dendrimers. While amphiphilic and self-assembling Janus dendrimers can form complexes large enough to increase their plasma circulation times, the drug loading of Janus dendrimers has been largely limited to use with drugs that can be chemically conjugated, which is the same weakness of dendrimers. Novel designs that allow for dendritic polymers to physically entrap chemically inert drug molecules, in addition to possible conjugation options, are an alternative to address this issue.

\subsection{Integration of Dendritic Polymers with Linear Polymers}

\subsubsection{Designing Amphiphilic Linear-Dendritic Block Copolymers-The size-}

related issues and limited versatility of dendrimers in terms of carrying chemically unreactive drug molecules can be overcome by engineered linear-dendritic block copolymers (LDBC) that combine a dendritic scaffold with one or more linear polymers (Figure 2).(49) Since their introduction by Frechét and co-workers in 1992, their multifunctional features helped them draw considerable attention.(50) Symmetrically branched dendrons possess a well-defined number of end groups that offer versatility to the macromolecules by applying a variety of surface chemistries and architectural engineering, which tunes their physiochemical and biological properties. For instance, the block copolymer design allows amphiphilic dendritic polymers to self-assemble into large micellar structures, extending their biomedical utilities (Figure 2a-c). In contrast to dendrimers, LDBC-based micelles can take advantage of the EPR effect due to their enlarged size and allow hydrophobic drug molecules to be stably encapsulated into their cores (Figure 3a). The Hammond group synthesized an amphiphilic PAMAM-poly(propylene oxide)-PAMAM triblock copolymer with dendrons ranging from G2 to G6 (Figure 2a).(51) These LDBCs were able to selfassemble into micelles (9-18 nm in diameter) with high drug encapsulation efficiencies greater than $80 \%$. Moreover, the LDBC micelles constructed with a dumbbell architecture of PAMAM-poly(caprolactone) (PCL)-PEG-PAMAM-PCL enhanced the drug loading efficiency by over $18 \%$ and prolonged the release rates of encapsulated DOX compared to linear micelles of a similar composition (Figure 2b).(52) The superior loading properties of the LDBC micelles was attributed to the dendritic architecture of the LBDC thermodynamically stabilizing the assembly.

Our group further investigated the role of the unique molecular architecture of LDBCs on their self-assembly. We conducted a systematic comparison of the self-assembly process of PEGylated dendron copolymers (PCL- $b$-polyester dendron-PEG) over their linear block copolymer counterparts at similar hydrophilic-lipophilic balances (HLBs) (Figure 2c).(53) The LDBC-based micelles displayed critical micelle concentrations as low as in the order of $10^{-8} \mathrm{M}$ that were 1-2 orders of magnitude lower than linear polymer (PCL- $b$-PEG)-based micelles at similar HLBs. This trend has also been observed in dendritic poly(lactide- $c o-$ glycolide) (PLGA)- $b$-dendron- $b$-PEG copolymers.(54) This series of results suggests that addition of the conically pre-organized dendritic architecture to the polymer architecture lowers the entropic cost during self-assembly, resulting in formation of thermodynamically 
stable micelle structures. Interestingly, the result of a molecular dynamic (MD) simulation revealed that the dendritic PEG architecture also allowed for a more completed PEG coverage over the hydrophobic core of the micelle, compared to linear block copolymerbased micelles (Figure 3b). $(53,55)$ Since the PEG chains form a hydrophilic stealth shell on the NP that reduces non-specific cellular interactions and protein adsorption, these results suggest that the LDBC micelles have potential to perform as an efficient nanocarrier platform with prolonged blood circulation and reduced off-targeting.

The superior thermodynamic stability of the LDBC micelles was further supported by our study comparing the premature release of cargo molecules from the LDBC and linear PCL$b$-PEG-based micelles. The LDBC micelles built using PCL- $b$-polyester dendron-PEG were able to prevent their hydrophobic cargo from being released in PBS for up to $48 \mathrm{~h}$ in the presence of cells whereas the linear PCL- $b$-PEG micelles released more than 2-times the cargo under the same conditions.(55) Similarly, LDBCs prepared with PCL of a molecular weight of $14 \mathrm{~kg} \cdot \mathrm{mol}^{-1}$ demonstrated a prolonged, controlled release of indomethacin compared to linear micelles with a similar composition.(53) We also demonstrated that charge-based cellular interactions of the LDBC micelles could be modulated by changing the length of the conjugated PEG chains. Non-specific cellular interactions and toxicities of positively charged LDBC micelles were only observed in the micelles with short PEG (molecular weight of $600 \mathrm{~g} / \mathrm{mol}$ ) outer layers, which were eliminated in the case of the micelles with the same charge but with a PEG of $2,000 \mathrm{~g} / \mathrm{mol}$. This phenomenon was explained by the formation of hydrogen bonds between the PEG backbone and terminal amine groups, resulting in back-folding of the charged end groups, which sequestered charge-dependent non-specific cellular interactions. This behavior was more pronounced by the longer PEG as the shorter PEG chains exhibited less hydrogen bond formation, thereby allowing the charged groups exposed on the micelles surface to interact with negatively charged cell membranes. Furthermore, terminal charges may also affect the ability of LDBCs to encapsulate small molecules. Our group previously compared the encapsulation efficiency of the LDBC-based micelles with amine (positively charged), carboxyl (negatively charged), and acetamide (charge neutral) terminal groups.(56) Interestingly, the carboxyl-terminated micelles exhibited significantly increased loading capacity of the anticancer drug endoxifen (EDX) over the other micelles by more than 5 times. Whereas $100 \%$ of EDX released from liposomes within the first $8 \mathrm{~h}$, an equivalent release from the LDBC micelle formulations did not occur until day 5. It is also noteworthy that the LDBC micelles were able to enhance the permeation of EDX across the mouse and human skin up to 20and 4-fold, respectively, compared to the ethanol control, demonstrating the potential of these LDBC in transdermal delivery as well.(56)

3.2.2 Linear-Dendritic Block Copolymer for Targeted Drug Delivery-One of the key advantages of dendritic structure is that it facilitates multivalent binding, the simultaneous binding of multiple ligands and their receptors in a highly localized area, resulting in a dramatic increase in binding constant. This phenomenon has been utilized to substantially increase the targeting efficacy of dendrimers conjugated with targeting agents. $(57,58)$ Integration of the dendritic architecture into polymeric micelles thus provides a platform for utilizing the multivalent properties of dendrimers, which typically results in 
orders of magnitude enhancement in binding constants. $(57,58)$ Poon et al. synthesized FAtargeted $\beta$-benzyl-L-aspartate-polyester-PEG LDBCs forming "patchy" micelles for enhanced targeting and plasma circulation (Figure 2d). $(59,60)$ They reported that LDBCs with $\sim 3.2 \mathrm{FA} /$ polymer mixed at a 60:40 ratio to untargeted polymers resulted in the highest binding avidity and cellular uptake of the resulting micelles by FA-receptor overexpressing cells. This strong multivalent ligand-receptor binding reportedly worked synergistically with the EPR effect to promote the tumor retention of the micelles.

LDBCs have also been largely used for constructing stimuli-responsive NPs for targeted drug delivery (Figure 2e). The well-defined nature of the dendron block enables fine-tuning of the material properties in a controlled and predictable way. The Frechét group first developed a pH-responsive LDBC-based micelle system by conjugating highly acid sensitive cyclic acetals to the periphery of polylysine and polyester dendrons with a PEO tail.(61) The micelles retained their hydrophobic cargos at $\mathrm{pH} 7.4$ and rapidly released the drug at $\mathrm{pH} 5$ due to the rapid hydrolysis of the acetal groups, which facilitates disintegration of the assembly. In a follow-up study, these micelles demonstrated the ability to encapsulate DOX and selectively release in acidic environments, such as the tumor tissue and endocytic vesicles (endosomes and lysosomes).(62) More recently, Harnoy et al. synthesized a series of LDBCs composed of an enzymatic-responsive hydrophobic dendron and linear PEG.(63) The $\mathrm{G} 2$ dendron block they used contained phenyl acetamide end groups that were cleaved by the enzyme penicillin G amidase. By tuning the length of the PEG chains, enzymeresponsive micelles with individualized degradation and disassembly rates were prepared, demonstrating their potential as modular nanocarriers that are stimuli responsive.

3.2.3 Linear-Dendritic Block Copolymers in Gene Delivery-In addition to forming micelles through self-assembly, LDBCs can be engineered to complex with nucleic acids to form "polyplexes." LDBCs containing a PEG conjugated to the core of a cationic dendron can form polyplexes that have surface coverage of PEG. This design may provide an approach to limiting the non-specific interactions seen with cationic dendrimers in gene delivery. Wood et al. compared the transfection efficiency of the commercially available polyethylenimine (PEI) and a range of the PAMAM-PEG-carbohydrate conjugates containing G0 to G6 PAMAMs (Figure 2f). Their results demonstrated that the polyplexes formed from LDBCs with G4-G6 exhibited better transfection efficiencies than PEI in serum-containing media.(64) Similarly, others have synthesized a series of methoxyl-PEGdendritic polyglycerol-graft-tris(2-aminoethyl)amine (mPEG-DPG- $g$-TAEA) with different numbers of TAEA arms to evaluate their ability to complex and deliver oligonucleotides

(Figure 2g).(65) They reported that the buffering capacity, size, and zeta potential of the complexes were tailored by varying the generation of the dendritic DPG and the degree of TAEA substitution. Because of the unique dendritic topology, the TAEA achieved strong DNA binding at relatively low amine densities, thereby allowing them to exhibit higher transfection efficiencies compared to PEI with a molecular weight of 5,000 g/mol (PEI5K), while maintaining significantly lower cytotoxicities. In addition, the conjugated PEG chain granted the LDBC polyplexes improved resistance to the adsorption of bovine serum albumin than PEI5K. These data suggest that the combination of linear-block copolymers 
and dendrimers results in the unique properties of LDBC, making them a promising platform for gene delivery.

\subsection{Hybridization of Dendrimers with Other Nanocarriers}

While LDBCs have demonstrated promise in designing better dendritic nanocarriers, they are unable to take advantage of the small, controlled size of dendrimers that typically results in enhanced tumor penetration and distinct biodistributions. In order to hybridize some of these traits with those of larger PEGylated NPs, a few hybrid NPs have been introduced in which dendrimers are encapsulated in larger NPs.(66-70) The larger NPs typically exhibit greater plasma circulation times and utilize the EPR effect leading to greater tumor accumulation by passive targeting. Upon reaching the tumor, release of the dendrimers would allow them to efficiently permeate the tumor mass and achieve enhanced active targeting via multivalent binding. With this idea, our group developed a hybrid NP platform consisting of FA-targeted G4 PAMAM dendrimers (G4-FA) encapsulated within a larger poly(ethylene glycol)- $b$-(D,L-lactide) (PEG-PLA) shell ( $100 \mathrm{~nm}$ in diameter), as illustrated in Figure 3c. $(66,68)$ The hybrid NPs displayed significantly enhanced circulation times compared to the free dendrimer conjugates, with biodistributions more like those of large NPs and less similar to free dendrimers. Moreover, the encapsulated dendrimers exhibited greater tumor accumulation in a FA-overexpressing tumor xenograft. These results are likely due to the larger polymeric NP shell reducing the rapid clearance that is typically observed in the case of dendrimers. Similarly, the G4-FA conjugates can take advantage of ligandmediated targeting and multivalent binding, further increasing overall targeting efficacy(68). Although the large NPs promote accumulation at the tumor site, the large size limits it from effective permeation through the tumor mass.(69) In contrast, small actively targeted dendrimers have been demonstrated to rapidly permeate in a multicellular tumor spheroid (MCTS) model (Figure 3c, bottom).(69) Additionally, the hybrid NP platform confers several other advantages in drug delivery. For instance, the kinetics of their release of the dendrimers and thereby the dendrimer-cell interactions can be controlled using polymeric shells with various PLA molecular weights.(67) The release profiles of dendrimers were inversely proportional to the molecular weight of the encapsulating polymers, with high molecular weight PLA ( $45 \mathrm{~kg} / \mathrm{mol}$ ) exhibiting substantially slower dendrimer release kinetics. These findings support that encapsulation of targeted dendrimers within larger PEG-PLA NPs confers increased plasma circulation and tumor accumulation, while also offering a way to control dendrimer release profiles.

Another example is dendrimer-lipid hybrid NPs. Sun et al. have reported the use of dendrimerlipid "nanoassemblies" to combine the properties of G6 polyaminoester dendrimers with $\mathrm{pH}$-dependent 2-(N,N-diethylamino)ethyl termini with lipid NPs by encapsulating the dendrimers in PEGylated liposomes for the enhanced biodistribution of DOX.(70) The DOX-loaded NPs formulated with dendrimers prolonged the drug release kinetics, compared to the dendrimer-free assemblies and DOX-loaded free dendrimers. Following incubation with SKOV-3 cells, the lipid components of the hybrid NPs were observed on the cell membrane whereas dendrimers were found within the cells, suggesting that their release from the nanoassemblies promoted cellular uptake for increased cytoplasmic DOX concentrations. Notably, the nanoassemblies displayed more stable 
circulation profiles and anti-tumor efficacy compared to linear polymer micelles composed of PCL-PEG. Moreover, the dendrimer-liposome hybrids demonstrated relatively homogeneous tumor distributions and efficient permeation into the tumor mass.

Although hybrid NPs present unique advantages, they still have their limitations. For instance, obtaining efficient drug loadings are difficult when encapsulating dendrimers within NPs due to their large molecular weights compared to free drugs. Moreover, larger NPs tend to be uptaken by the organs of the RES system, causing them to preferentially accumulate in the liver and spleen. Nonetheless, hybrid NPs offers unique advantages and a viable option to integrate advantageous characteristics of multi-scaled nanomaterials, which is one of the promising approaches to design improved nanocarriers.

\subsection{Conclusion}

Although intensive efforts have been put forward to the use of dendrimers as a nanocarrier platform particularly for targeting cancer, dendrimer progression to the clinical arena has been slow. In tumor treatment, there are several unique barriers that block NPs from efficiently reaching the tumor and evoking a desirable therapeutic action in a precisely controlled manner. Although dendrimers have advantageous traits for overcoming these barriers, they are limited by their short circulation times, small size and inability to passively target tumors, and poor drug loadings. As a result, a number of approaches to improve the delivery efficacy of dendrimers have been attempted. Among those, three approaches are highlighted in this review paper, each with distinct advantages and disadvantages. Firstly, modifying the dendrimer structure and surface is a relatively easy method for overcoming undesired cell interactions and poor circulation abilities; however, simple modifications do not significantly enhance tumor targeting or increase loading capabilities. Secondly, integration of the dendrons into LDBCs to form self-assembled micelles has shown promising results in increasing the loading and size to be enough for passive targeting. These benefits however come at the cost of efficient tumor penetration that is often observed by small dendrimers. Alternatively, incorporation of the dendrimers into larger PEGylated NPs has demonstrated the potential to overcome these barriers, but with significantly added material complexities. All in all, it is necessary that the most appropriate approach should be chosen based on individual cases and characteristics of each tumor. It is becoming obvious that there is no one platform technology that solves all issues. Instead, a sophisticated tailoring of nanocarriers with patient-specific therapeutic agents/methods would be required to achieve truly personalized medicine in years to come. Although these intricacies raise concerns for large scale preparations, it appears that they are necessary for overcoming the complex biological barriers for the treatment of cancer.

\section{Acknowledgements}

This work was supported by National Science Foundation (NSF) under grant \# DMR1409161, National Cancer Institute, NIH under grant \# 1R01CA182528-01, and the Technological Innovation R\&D Program (grant \# S2083505) funded by the Small and Medium Business Administration of Korea. 


\section{References}

1. Siegel R, Ma J, Zou Z, Jemal A. Cancer statistics, 2014. CA: A Cancer Journal for Clinicians. 2014; 64(1):9-29. [PubMed: 24399786]

2. Peer D, Karp JM, Hong S, Farokhzad OC, Margalit R, Langer R. Nanocarriers as an emerging platform for cancer therapy. Nature Nanotech. 2007; 2(12):751-60.

3. Pearson RM, Hsu H-j, Bugno J, Hong S. Understanding nano-bio interactions to improve nanocarriers for drug delivery. MRS Bulletin. 2014; 39(03):227-37.

4. Pearson RM, Juettner V, Hong S. Biomolecular Corona on Nanoparticles: A Survey of Recent Literature and its Implications in Targeted Drug Delivery. Front Chem. 2014; 2:108. [PubMed: 25506050]

5. Kim BYS, Rutka JT, Chan WCW. Nanomedicine. New England Journal of Medicine. 2010; 363(25):2434-43. [PubMed: 21158659]

6. Marin E, Briceño MI, Caballero-George C. Critical evaluation of biodegradable polymers used in nanodrugs. International journal of nanomedicine. 2013; 8:3071-91. [PubMed: 23990720]

7. Liechty WB, Kryscio DR, Slaughter BV, Peppas NA. Polymers for Drug Delivery Systems. Annual review of chemical and biomolecular engineering. 2010; 1:149-73.

8. Gref R, Minamitake Y, Peracchia M, Trubetskoy V, Torchilin V, Langer R. Biodegradable longcirculating polymeric nanospheres. Science. 1994; 263:1600-3. [PubMed: 8128245]

9. Langer R. New methods of drug delivery. Science. 1990; 249(4976):1527-33. [PubMed: 2218494]

10. Langer R, Tirrell DA. Designing materials for biology and medicine. Nature. 2004; 428(6982): 487-92. [PubMed: 15057821]

11. Bourzac K. Nanotechnology: Carrying drugs. Nature. 2012; 491:S58-S60. [PubMed: 23320289]

12. Duncan R. Polymer conjugates as anticancer nanomedicines. Nature reviews Cancer. 2006; 6(9): 688-701. [PubMed: 16900224]

13. Duncan R. The dawning era of polymer therapeutics. Nat Rev Drug Discov. 2003; 2(5):347-60. [PubMed: 12750738]

14. Bugno J, Hsu H-j, Hong S. Recent advances in targeted drug delivery approaches using dendritic polymers. Biomater Science. 2015 10.1039/C4BM00351A.

15. Soleyman R, Adeli M. Impact of dendritic polymers on nanomaterials. Polym Chem. 2015; 6:1024.

16. Sowinska M, Urbanczyk-Lipkowska Z. Advances in the chemistry of dendrimers. New Journal of Chemistry. 2014; 38(6):2168-203.

17. Esfand R, Tomalia DA. Poly(amidoamine) (PAMAM) dendrimers: from biomimicry to drug delivery and biomedical applications. Drug Discovery Today. 2001; 6(8):427-36. [PubMed: 11301287]

18. Buhleier E, Wehner W, VÖGtle F. "Cascade"- and "Nonskid-Chain-like" Syntheses of Molecular Cavity Topologies. Synthesis. 1978; (02):155-8.

19. Tomalia DA, Baker H, Dewald J, Hall M, Kallos G, Martin S, et al. A New Class of Polymers: Starburst-Dendritic Macromolecules. Polym J. 1985; 17(1):117-32.

20. Hawker CJ, Frechet JMJ. Preparation of polymers with controlled molecular architecture. A new convergent approach to dendritic macromolecules. Journal of the American Chemical Society. 1990; 112(21):7638-47.

21. van Dongen MA, Desai A, Orr BG, Baker JR Jr, Banaszak Holl MM. Quantitative analysis of generation and branch defects in G5 poly(amidoamine) dendrimer. Polymer. 2013; 54(16):412633. [PubMed: 24058210]

22. Mullen DG, Borgmeier EL, Desai AM, van Dongen MA, Barash M, Cheng X-m, et al. Isolation and Characterization of Dendrimers with Precise Numbers of Functional Groups. Chemistry - A European Journal. 2010; 16(35):10675-8.

23. Mullen DG, Fang M, Desai A, Baker JR, Orr BG, Banaszak Holl MM. A Quantitative Assessment of Nanoparticle-Ligand Distributions: Implications for Targeted Drug and Imaging Delivery in Dendrimer Conjugates. ACS Nano. 2010; 4(2):657-70. [PubMed: 20131876] 
24. van Dongen MA, Silpe JE, Dougherty CA, Kanduluru AK, Choi SK, Orr BG, et al. Avidity Mechanism of Dendrimer-Folic Acid Conjugates. Molecular Pharmaceutics. 2014; 11(5):1696706. [PubMed: 24725205]

25. Kesharwani P, Jain K, Jain NK. Dendrimer as nanocarrier for drug delivery. Progress in Polymer Science. 2014; 39(2):268-307.

26. Percec V, Cho WD, Mosier PE, Ungar G, Yeardley DJP. Structural Analysis of Cylindrical and Spherical Supramolecular Dendrimers Quantifies the Concept of Monodendron Shape Control by Generation Number. Journal of the American Chemical Society. 1998; 120(43):11061-70.

27. Maiti PK, Çağın T, Wang G, Goddard WA. Structure of PAMAM Dendrimers: Generations 1 through 11. Macromolecules. 2004; 37(16):6236-54.

28. Hawker CJ, Farrington PJ, Mackay ME, Wooley KL, Frechet JMJ. Molecular Ball Bearings: The Unusual Melt Viscosity Behavior of Dendritic Macromolecules. Journal of the American Chemical Society. 1995; 117(15):4409-10.

29. Uppuluri S, Keinath SE, Tomalia DA, Dvornic PR. Rheology of Dendrimers. I. Newtonian Flow Behavior of Medium and Highly Concentrated Solutions of Polyamidoamine (PAMAM) Dendrimers in Ethylenediamine (EDA) Solvent. Macromolecules. 1998; 31(14):4498-510.

30. Kaminskas LM, McLeod VM, Porter CJH, Boyd BJ. Association of Chemotherapeutic Drugs with Dendrimer Nanocarriers: An Assessment of the Merits of Covalent Conjugation Compared to Noncovalent Encapsulation. Molecular Pharmaceutics. 2012; 9(3):355-73. [PubMed: 22250750]

31. Satsangi A, Roy SS, Satsangi RK, Vadlamudi RK, Ong JL. Design of a Paclitaxel prodrug conjugate for active targeting of an enzyme upregulated in breast cancer cells. Molecular Pharmaceutics. 2014; 11(6):1906-18. [PubMed: 24847940]

32. Patri AK, Kukowska-Latallo JF, Baker JR Jr. Targeted drug delivery with dendrimers: Comparison of the release kinetics of covalently conjugated drug and non-covalent drug inclusion complex. Advanced Drug Delivery Reviews. 2005; 57(15):2203-14. [PubMed: 16290254]

33. Hong S, Bielinska AU, Mecke A, Keszler B, Beals JL, Shi X, et al. Interaction of poly(amidoamine) dendrimers with supported lipid bilayers and cells: hole formation and the relation to transport. Bioconjugate Chemistry. 2004; 15(4):774-82. [PubMed: 15264864]

34. Hong S, Leroueil PR, Janus EK, Peters JL, Kober M-M, Islam MT, et al. Interaction of Polycationic Polymers with Supported Lipid Bilayers and Cells: Nanoscale Hole Formation and Enhanced Membrane Permeability. Bioconjugate Chemistry. 2006; 17(3):728-34. [PubMed: 16704211]

35. Malik N, Wiwattanapatapee R, Klopsch R, Lorenz K, Frey H, Weener JW, et al. Dendrimers:: Relationship between structure and biocompatibility in vitro, and preliminary studies on the biodistribution of 125I-labelled polyamidoamine dendrimers in vivo. Journal of Controlled Release. 2000; 65(1-2):133-48. [PubMed: 10699277]

36. Kobayashi H, Kawamoto S, Saga T, Sato N, Hiraga A, Konishi J, et al. Micro-MR angiography of normal and intratumoral vessels in mice using dedicated intravascular MR contrast agents with high generation of polyamidoamine dendrimer core: Reference to pharmacokinetic properties of dendrimer-based MR contrast agents. Journal of Magnetic Resonance Imaging. 2001; 14(6):70513. [PubMed: 11747027]

37. Kobayashi H, Sato N, Hiraga A, Saga T, Nakamoto Y, Ueda H, et al. 3D-micro-MR angiography of mice using macromolecular MR contrast agents with polyamidoamine dendrimer core with reference to their pharmacokinetic properties. Magnetic Resonance in Medicine. 2001; 45(3):45460. [PubMed: 11241704]

38. Kukowska-Latallo JF, Candido KA, Cao Z, Nigavekar SS, Majoros IJ, Thomas TP, et al. Nanoparticle Targeting of Anticancer Drug Improves Therapeutic Response in Animal Model of Human Epithelial Cancer. Cancer Research. 2005; 65(12):5317-24. [PubMed: 15958579]

39. Wu W, Driessen W, Jiang X. Oligo(ethylene glycol)-Based Thermosensitive Dendrimers and Their Tumor Accumulation and Penetration. Journal of the American Chemical Society. 2014; 136(8): 3145-55. [PubMed: 24506735]

40. Yang Y, Sunoqrot S, Stowell C, Ji J, Lee C-W, Kim JW, et al. Effect of Size, Surface Charge, and Hydrophobicity of Poly(amidoamine) Dendrimers on Their Skin Penetration. Biomacromolecules. 2012; 13(7):2154-62. [PubMed: 22621160] 
41. Kaminskas LM, Boyd BJ, Karellas P, Henderson SA, Giannis MP, Krippner GY, et al. Impact of Surface Derivatization of Poly-1-lysine Dendrimers with Anionic Arylsulfonate or Succinate Groups on Intravenous Pharmacokinetics and Disposition. Molecular Pharmaceutics. 2007; 4(6): 949-61. [PubMed: 17953445]

42. Caminade A-M, Laurent R, Delavaux-Nicot B, Majoral J-P. “Janus” dendrimers: syntheses and properties. New J Chem. 2012; 36:217-26.

43. Wooley KL, Hawker CJ, Frechet JMJ. Polymers with controlled molecular architecture: control of surface functionality in the synthesis of dendritic hyperbranched macromolecules using the convergent approach. Journal of the Chemical Society, Perkin Transactions 1. 1991; (5):1059-76.

44. Aoi K, Itoh K, Okada M. Divergent/Convergent Joint Approach with a Half-Protected Initiator Core To Synthesize Surface-Block Dendrimers. Macromolecules. 1997; 30(25):8072-4.

45. Imae T, Ito M, Aoi K, Tsutsumiuchi K, Noda H, Okada M. Formation of organized adsorption layers by amphiphilic dendrimers. Colloids and Surfaces A: Physicochemical and Engineering Aspects. 2000; 175(1-2):225-34.

46. Lee CC, Gillies ER, Fox ME, Guillaudeu SJ, Fréchet JMJ, Dy EE, et al. A single dose of doxorubicin-functionalized bow-tie dendrimer cures mice bearing C-26 colon carcinomas. Proceedings of the National Academy of Sciences. 2006; 103(45):16649-54.

47. Al-Jamal KT, Ramaswamy C, Florence AT. Supramolecular structures from dendrons and dendrimers. Advanced Drug Delivery Reviews. 2005; 57(15):2238-70. [PubMed: 16310885]

48. Bayele HK, Sakthivel T, O'Donell M, Pasi KJ, Wilderspin AF, Lee CA, et al. Versatile peptide dendrimers for nucleic acid delivery. Journal of Pharmaceutical Sciences. 2005; 94(2):446-57. [PubMed: 15614813]

49. Wurm F, Frey H. Linear-dendritic block copolymers: The state of the art and exciting perspectives. Prog Polym Sci. 2011; 36(1):1-52.

50. Gitsov I, Wooley KL, Fréchet JMJ. Novel Polyether Copolymers Consisting of Linear and Dendritic Blocks. Angew Chem Int Ed. 1992; 31(9):1200-2.

51. Nguyen PM, Hammond PT. Amphiphilic Linear-Dendritic Triblock Copolymers Composed of Poly(amidoamine) and Poly(propylene oxide) and Their Micellar-Phase and Encapsulation Properties. Langmuir. 2006; 22(18):7825-32. [PubMed: 16922570]

52. Yang Y, Hua C, Dong C-M. Synthesis, Self-Assembly, and In Vitro Doxorubicin Release Behavior of Dendron-like/Linear/Dendron-like Poly( $\varepsilon$-caprolactone)-b-Poly(ethylene glycol)-b-Poly( $\varepsilon$ caprolactone) Triblock Copolymers. Biomacromolecules. 2009; 10(8):2310-8. [PubMed: 19618927]

53. Bae JW, Pearson RM, Patra N, Sunoqrot S, Vukovic L, Kral P, et al. Dendron-mediated selfassembly of highly PEGylated block copolymers: a modular nanocarrier platform. Chemical Communications. 2011; 47(37):10302-4. [PubMed: 21858356]

54. Santos JL, Herrera-Alonso M. Kinetically Arrested Assemblies of Architecturally Distinct Block Copolymers. Macromolecules. 2014; 47(1):137-45.

55. Hsu, H-j; Sen, S.; Pearson, RM.; Uddin, S.; Král, P.; Hong, S. Poly(ethylene glycol) Corona Chain Length Controls End-Group-Dependent Cell Interactions of Dendron Micelles. Macromolecules. 2014

56. Yang Y, Pearson RM, Lee O, Lee C-W, Chatterton RT, Khan SA, et al. Dendron-Based Micelles for Topical Delivery of Endoxifen: A Potential Chemo-Preventive Medicine for Breast Cancer. Advanced Functional Materials. 2014; 24(17):2442-9.

57. Hong S, Leroueil PR, Majoros IJ, Orr BG, Baker JR Jr, Banaszak Holl MM. The Binding Avidity of a Nanoparticle-Based Multivalent Targeted Drug Delivery Platform. Chemistry \& Biology. 2007; 14(1):107-15. [PubMed: 17254956]

58. Myung JH, Gajjar KA, Saric J, Eddington DT, Hong S. Dendrimer-Mediated Multivalent Binding for the Enhanced Capture of Tumor Cells. Angewandte Chemie International Edition. 2011; 50(49):11769-72.

59. Poon Z, Chen S, Engler AC, Lee H-i, Atas E, von Maltzahn G, et al. Ligand-Clustered "Patchy" Nanoparticles for Modulated Cellular Uptake and In Vivo Tumor Targeting. Angewandte Chemie International Edition. 2010; 49(40):7266-70. 
60. Poon Z, Lee JA, Huang S, Prevost RJ, Hammond PT. Highly stable, ligand-clustered "patchy" micelle nanocarriers for systemic tumor targeting. Nanomedicine: Nanotechnology, Biology and Medicine. 2011; 7(2):201-9.

61. Gillies ER, Jonsson TB, Fréchet JMJ. Stimuli-Responsive Supramolecular Assemblies of LinearDendritic Copolymers. Journal of the American Chemical Society. 2004; 126(38):11936-43. [PubMed: 15382929]

62. Gillies ER, Fréchet JMJ. pH-Responsive Copolymer Assemblies for Controlled Release of Doxorubicin. Bioconjugate Chemistry. 2005; 16(2):361-8. [PubMed: 15769090]

63. Harnoy AJ, Rosenbaum I, Tirosh E, Ebenstein Y, Shaharabani R, Beck R, et al. EnzymeResponsive Amphiphilic PEG-Dendron Hybrids and Their Assembly into Smart Micellar Nanocarriers. Journal of the American Chemical Society. 2014; 136(21):7531-4. [PubMed: 24568366]

64. Wood KC, Little SR, Langer R, Hammond PT. A Family of Hierarchically Self-Assembling Linear-Dendritic Hybrid Polymers for Highly Efficient Targeted Gene Delivery. Angewandte Chemie International Edition. 2005; 44(41):6704-8.

65. Yang B, Sun Y-x, Yi W-j, Yang J, Liu C-w, Cheng H, et al. A linear-dendritic cationic vector for efficient DNA grasp and delivery. Acta Biomaterialia. 2012; 8(6):2121-32. [PubMed: 22370448]

66. Sunoqrot S, Bae JW, Jin S-E, M. Pearson R, Liu Y, Hong S. Kinetically Controlled Cellular Interactions of Polymer-Polymer and Polymer-Liposome Nanohybrid Systems. Bioconjugate Chemistry. 2011; 22(3):466-74. [PubMed: 21344902]

67. Sunoqrot S, Bae JW, Pearson RM, Shyu K, Liu Y, Kim D-H, et al. Temporal Control over Cellular Targeting through Hybridization of Folate-targeted Dendrimers and PEGPLA Nanoparticles. Biomacromolecules. 2012; 13(4):1223-30. [PubMed: 22439905]

68. Sunoqrot S, Bugno J, Lantvit D, Burdette JE, Hong S. Prolonged blood circulation and enhanced tumor accumulation of folate-targeted dendrimer-polymer hybrid nanoparticles. Journal of Controlled Release. 2014; (0)

69. Sunoqrot S, Liu Y, Kim D-H, Hong S. In Vitro Evaluation of Dendrimer-Polymer Hybrid Nanoparticles on Their Controlled Cellular Targeting Kinetics. Molecular Pharmaceutics. 2013; 10(6):2157-66. [PubMed: 23234605]

70. Sun Q, Sun X, Ma X, Zhou Z, Jin E, Zhang B, et al. Integration of Nanoassembly Functions for an Effective Delivery Cascade for Cancer Drugs. Advanced Materials. 2014; 26(45):7615-21. [PubMed: 25328159] 


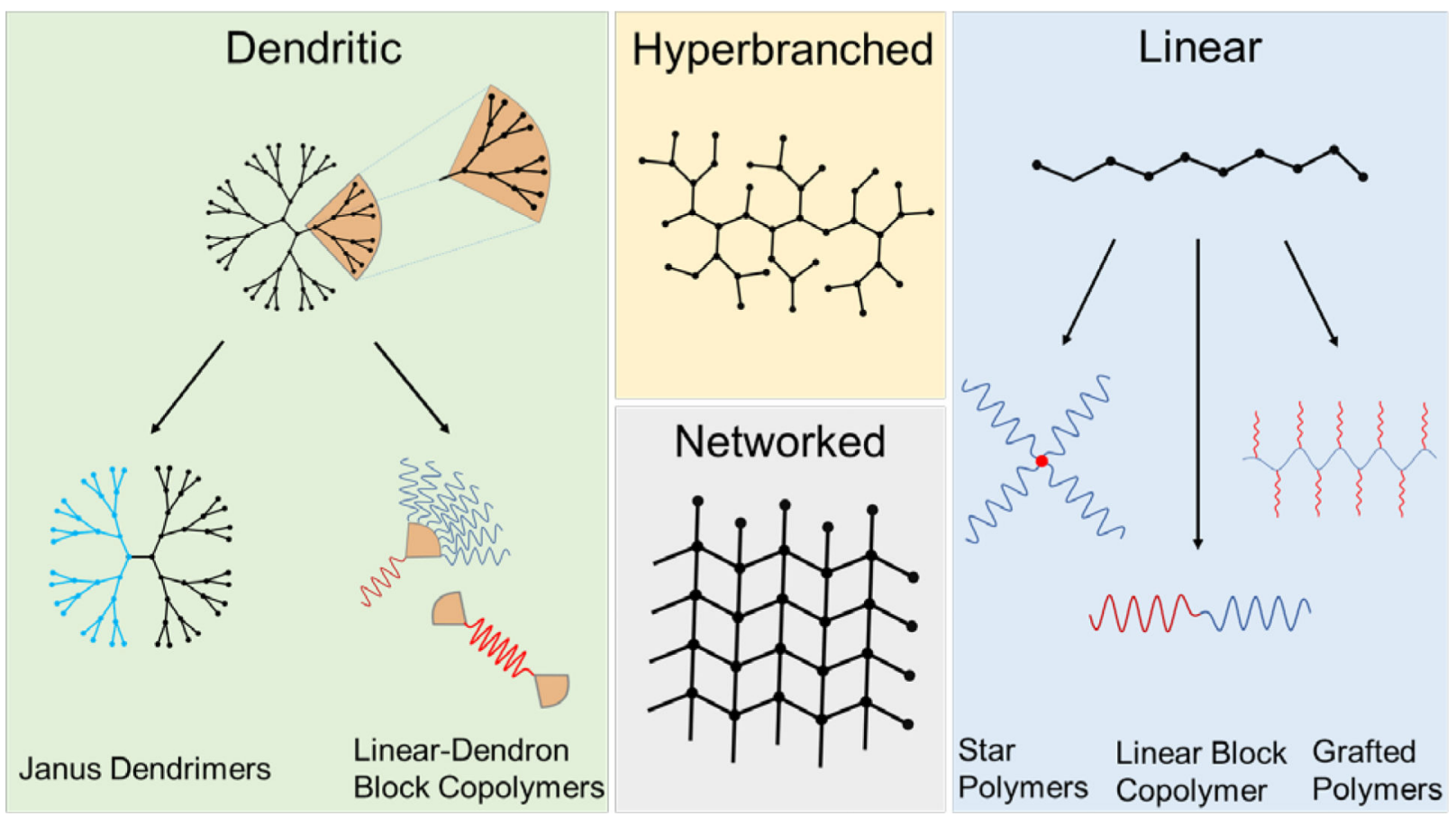

Figure 1.

Various polymeric architectures. Dendritic polymers include dendrimers and dendrons, which can be used to prepare integrated architectures such as Janus dendrimers and lineardendron block copolymers. Hyperbranched polymers have a high degree of random branching, in contrast to networked polymers that exhibit a highly ordered, cross-linked matrix. Linear polymers can be prepared to generate a wide range of architectures, including star and grafted polymers as well as linear block copolymers. 


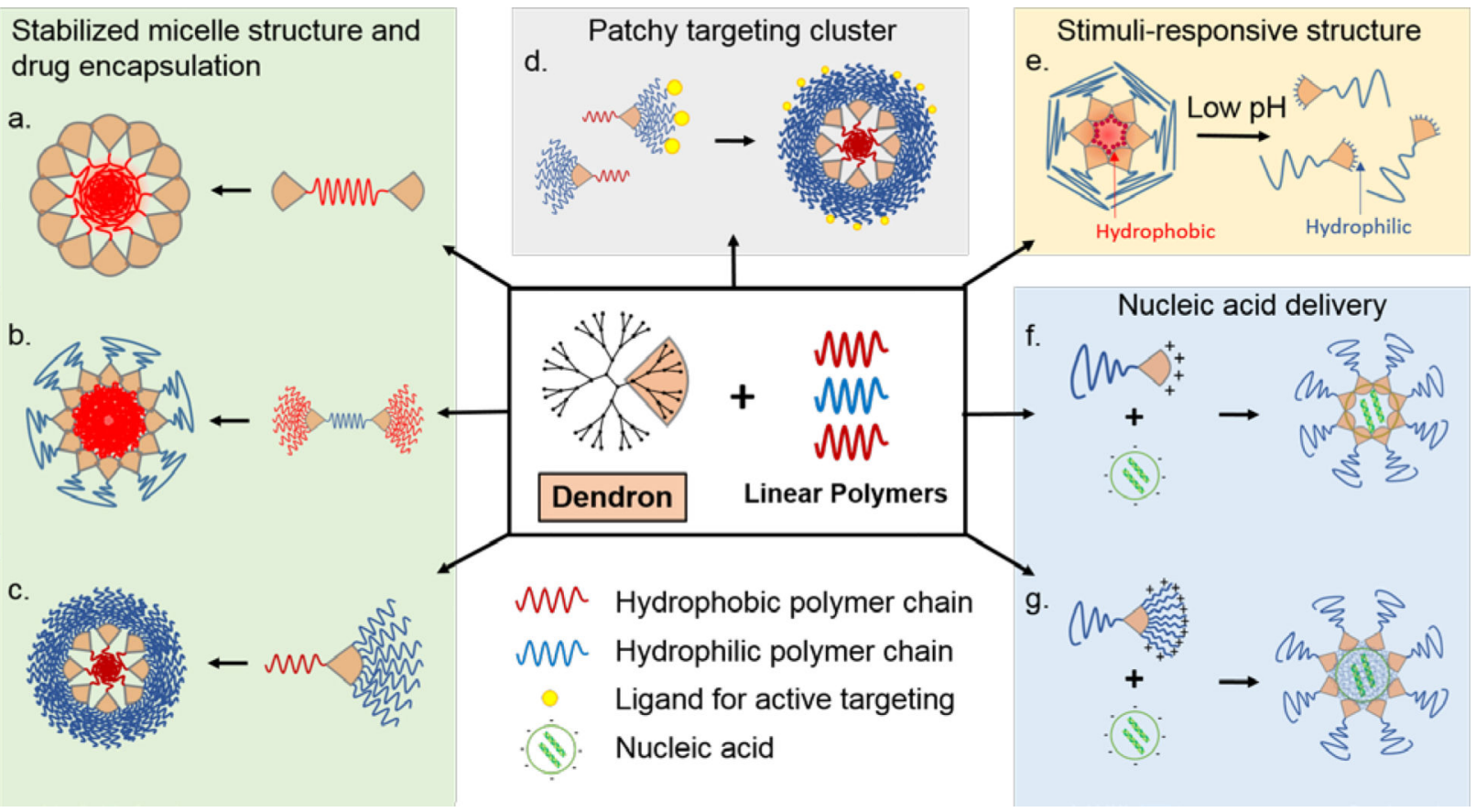

Figure 2.

Designs of linear-dendritic block copolymers. a-c) amphiphilic LDBCs for enhanced micelle stability and drug encapsulation: dendrons can be used as a hydrophilic block for conjugating with a hydrophobic linear polymer, or as a linker to connect multiple hydrophobic linear polymers to single hydrophilic linear polymer, and vice versa. d) A dendron-mediated patchy targeting cluster to increase targeting efficiency; e) stimuliresponsive LDBC-micelles for stimuli-responsive drug release; $\mathrm{f}$-g) LDBCs using dendritic topology to promote cationic polymer-nucleic acid complexation: either cationic dendrons or cationic linear polymer-conjugated dendrons can be used. 
a.

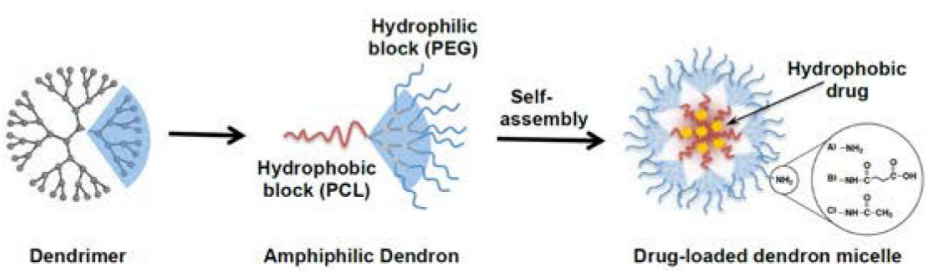

b.
C.

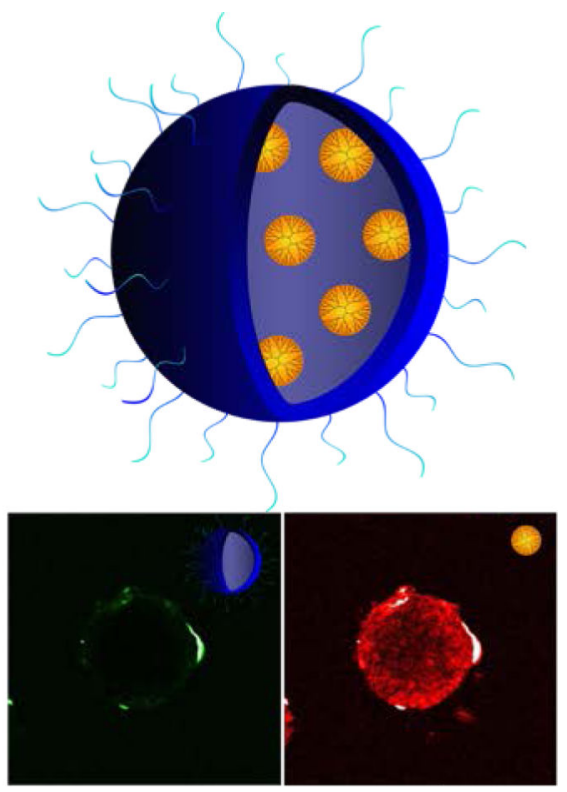

Figure 3.

Approaches to overcoming dendrimer limitations. a) Amphiphilic LDBCs self-assemble into a micelle with a dense coating of the hydrophilic, non-fouling polymer PEG. Hydrophobic drugs can be loaded within the core of the LDBC micelle, achieving higher loading efficiencies compared to dendrimers. b) An LDBC micelle displays greater surface coverage of PEG (red) compared to a linear polymer-based micelle exposing less of the hydrophobic core (blue) to aqueous solvent due to a controlled conical architecture of the dendron. c) A hybrid NP with encapsulated dendrimers significantly improves the plasma circulation times compared to free dendrimers. Moreover, dendrimers encapsulated within the NP permeate a multicellular tumor spheroid (bottom red) at a significantly higher efficiency upon release, compared to the large NP shell (bottom green). Reproduced from Ref. 13 with permission from The Royal Society of Chemistry. 